\title{
Article \\ A Priority-Based Autonomous Intersection Management (AIM) Scheme for Connected Automated Vehicles (CAVs)
}

\author{
Hui Zhang ${ }^{1} \mathbb{D}$, Rongqing Zhang ${ }^{2}$, Chen Chen ${ }^{1}$, Dongliang Duan ${ }^{3, * \mathbb{D}}$, Xiang Cheng ${ }^{1}$ and Liuqing Yang ${ }^{4}$ \\ 1 State Key Laboratory of Advanced Optical Communication Systems and Networks, Department of \\ Electronics, School of Electronics Engineering and Computer Science, Peking University, \\ Beijing 100871, China; huizhang1002@pku.edu.cn (H.Z.); c.chen@pku.edu.cn (C.C.); \\ xiangcheng@pku.edu.cn (X.C.) \\ 2 School of Software Engineering, Tongji University, Shanghai 200092, China; rongqingz@tongji.edu.cn \\ 3 Department of Electrical and Computer Engineering, University of Wyoming, Laramie, WY 82071, USA \\ 4 Department of Electrical and Computer Engineering, University of Minnesota, Minneapolis, MN 55455, USA; \\ qingqing@umn.edu \\ * Correspondence: dduan@uwyo.edu
}

Citation: Zhang, H.; Zhang, R.; Chen, C.; Duan, D.; Cheng, X.; Yan, L. A Priority-Based Autonomous Intersection Management (AIM) Scheme for Connected Automated Vehicles (CAVs). Vehicles 2021, 3, 533-544. https://doi.org/ 10.3390/vehicles3030032

Academic Editor: Yongzhi Zhang

Received: 9 July 2021

Accepted: 10 August 2021

Published: 13 August 2021

Publisher's Note: MDPI stays neutral with regard to jurisdictional claims in published maps and institutional affiliations.

Copyright: (c) 2021 by the authors. Licensee MDPI, Basel, Switzerland. This article is an open access article distributed under the terms and conditions of the Creative Commons Attribution (CC BY) license (https:/ / creativecommons.org/licenses/by/ $4.0 /)$.

\begin{abstract}
In this paper, we investigate the intersection traffic management for connected automated vehicles (CAVs). In particular, a decentralized autonomous intersection management scheme that takes into account both the traffic efficiency and scheduling flexibility is proposed, which adopts a novel intersection-vehicle model to check conflicts among CAVs in the entire intersection area. In addition, a priority-based collision-avoidance rule is set to improve the performance of traffic efficiency and shorten the delays of emergency CAVs. Moreover, a multi-objective function is designed to obtain the optimal trajectories of CAVs, which considers ride comfort, velocities of CAVs, fuel consumption, and the constraints of safety, velocity, and acceleration. Simulation results demonstrate that our proposed scheme can achieve good performance in terms of traffic efficiency and shortening the delays of emergency CAVs.
\end{abstract}

Keywords: autonomous intersection management; connected automated vehicles; crossing priority; optimal trajectory

\section{Introduction}

Intersection is an important scenario in transportation systems. As vehicles from different directions merge at intersections, traffic congestion or accidents happen easily. According to a survey, many wastes of fuel, time and economy were related to intersection congestions and accidents [1]. Therefore, lots of works about intersection management have been carried out to reduce these wastes over the years, such as optimal traffic light scheduling [2,3], cooperative autonomous driving [4,5] and so on.

Cooperative autonomous driving is one of the most promising methods for intersection management, which is the combination of vehicle-to-everything (V2X) [6] communications and connected automated vehicles (CAVs) [7] technologies. V2X is a typical application of the Internet of Things (IoT) [8] in the field of the intelligent transportation systems (ITS) [9], which integrates various wireless communication technologies to facilitate information exchange among vehicles, roadside infrastructure, pedestrians, and all other entities. With the help of V2X, CAVs can effectively sense and analyze the traffic environment in real time. Thus, CAVs can make optimal trajectories in advance to drive through intersections safely and efficiently. The application of cooperative autonomous driving at intersections is termed as autonomous intersection management (AIM), which can greatly improve the traffic efficiency and safety at intersections [10].

The research in AIM can be categorized into centralized and decentralized approaches [11]. The centralized approach [12] involves a management agent to coordinate the rights-of-way for the CAVs, where the management agent tries to optimize the system performance after 
receiving the information from all the related CAVs. In addition, the CAVs need to strictly follow the orders received from the management agent. The centralized approach could achieve better performance of traffic efficiency with the sacrifice of scheduling flexibility. In contrast, with the decentralized approach [13], each CAV determines its own control policy based on the information received from other CAVs and roadside infrastructure. Although it is difficult to achieve global optimization due to the lack of overall cooperation among CAVs, the decentralized approach has high flexibility and does not need an extra management agent. Therefore, lots of works have investigated the decentralized approach The authors in [13] designed a decentralized model predictive controller (MPC) to control each CAV crossing the intersection area, where a multi-objective function that considers rear-end collision avoidance at control zone and conflict avoidance at intersection zone is set to improve the performance of traffic efficiency, fuel consumption and ride comfort. L. Makarem and D. Gillet [14] introduced a new decentralized navigation function based on time-to-collision to improve energy consumption and motion smoothness, and showed that the proposed method achieved a significant performance gain over the traffic light scheme. In [15], a decentralized nonlinear model predictive control (NMPC) is proposed to coordinate the traffic flow of the CAVs, where the trajectory of CAV is obtained by optimizing a quadratic cost function and the crossing priorities are determined by the predicted arrival time or the First-Come First-Served (FCFS) rule. And H. Wei et al. [16] adopted game theory to avoid predicted collision inside the intersection zone, where a platoon structure formation algorithm was proposed to form coalitions of CAVs as players of the game.

In summary, the existing works on decentralized AIM are mainly on improving the traffic efficiency at intersections and ensuring safety when the CAVs are in the intersection zone. However, the priorities of emergency CAVs and the potential collisions after the CAVs leave the intersection zone were not considered. In this paper, we propose a decentralized AIM to address these issues. First, a priority-based collision-avoidance rule is proposed to improve the performance of traffic efficiency and delays of emergency CAVs. The rule, which considers the arrival time of CAVs to the intersection zone and the emergency level of CAVs, guarantees that the CAVs followed by emergency CAVs and the CAVs that can arrive at intersection zone earlier have higher crossing priorities. Moreover, to ensure the safety of CAVs in the entire intersection area, a novel intersection-vehicle model is proposed, which adopts conflict points to check conflicts in the intersection area and introduces collision check points to check conflicts after that CAVs leave the intersection. Then, the optimal trajectory planning of CAV is obtained by optimizing a multi-objective function, which takes into account the ride comfort, fuel consumption, velocities of CAVs, and the constraints of safety, velocity and acceleration.

The remainder of this paper is organized as follows. Section 2 presents the intersection-vehicle model. In Section 3, we propose a priority-based rule to avoid collisions between CAVs. Then, Section 4 analyzes the optimal trajectory of CAV. Simulation results are presented and evaluated in Section 5. Finally, some concluding remarks are presented in Section 4.

\section{System Model}

\subsection{Intersection Layout}

In this paper, we consider a typical intersection scenario where the intersection is connected with four bidirectional links with two lanes. The intersection area is divided into four zones as shown in Figure 1. In the running direction, the first zone far from the intersection is known as the far zone, which is used to adjust the crossing priorities of CAVs. After piloting into far zone, each CAV needs to share its driving information and optimize its trajectory planning while considering potential collisions with other CAVs that have higher crossing priorities. The second zone adjacent to the intersection is called the near zone, where the crossing priorities and the trajectory planning of CAVs do not change any more unless emergency situation happens. The area that two lanes intersect is termed as 
the intersection zone (IZ), and there are 20 conflict points in the IZ as illustrated in Figure 2. Finally, the zone that CAVs leave away the intersection is termed as the potential collision zone (PCZ), where the CAVs with the same destination have the possibilities to collide. In addition, the end of PCZ is called the collision check point, which is used to check the potential collision that the faster CAV cannot decelerate to avoid slower CAV in this zone.

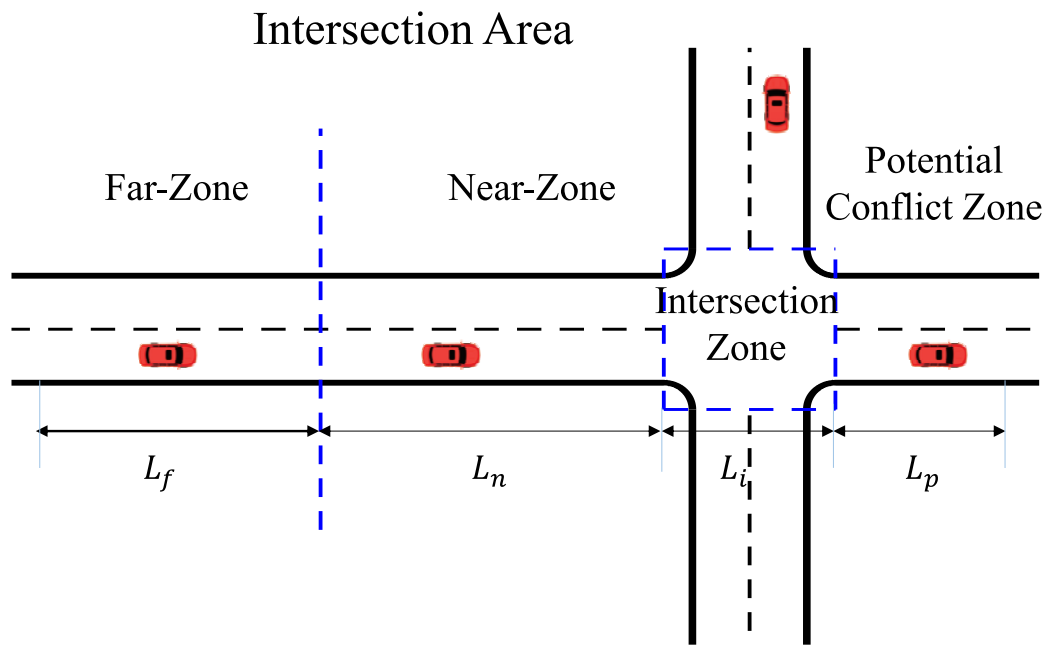

Figure 1. Intersection layout.

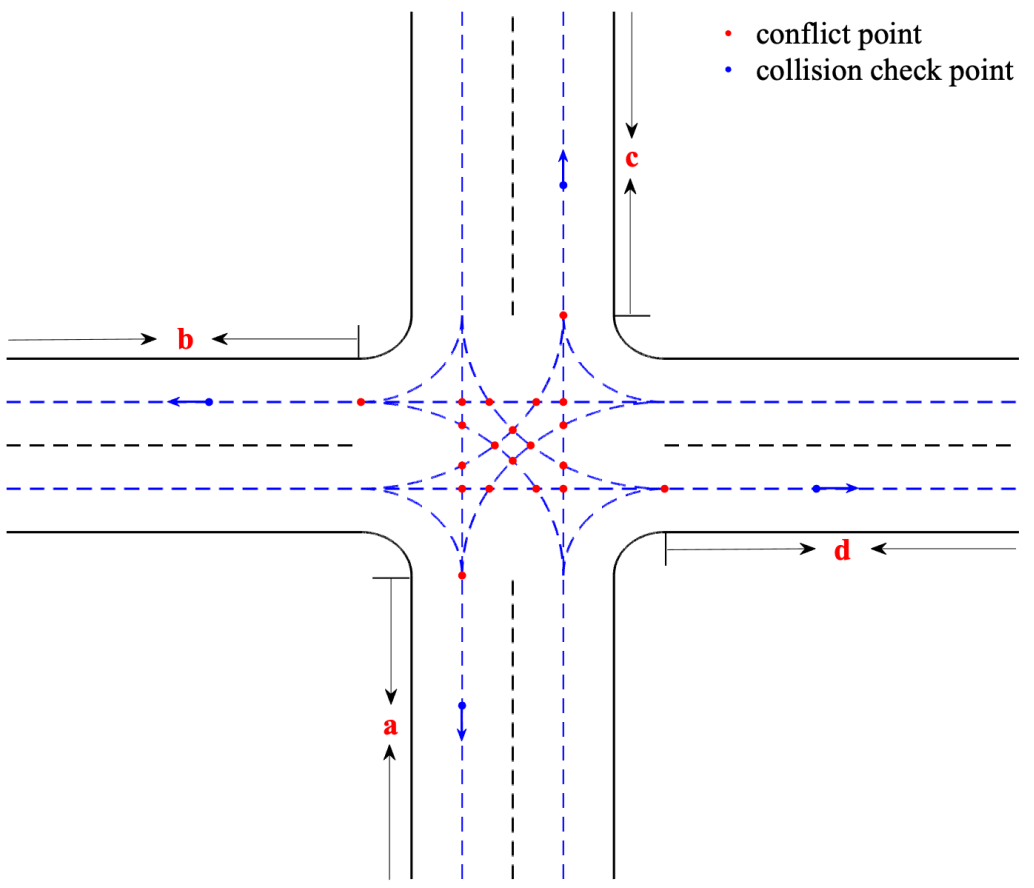

Figure 2. Conflict model at intersections.

\subsection{Vehicle Model}

The primary task of AIM is to guide the CAVs to pass the intersection safely and efficiently. Therefore, the CAVs need to control their longitudinal velocities to adjust the arrival time to the different positions on their driving trajectories, which guarantees that different CAVs cannot arrive at the same position at the same time. Thus, only the longitudinal kinematics of CAVs are considered in this paper.

To calculate the arrival time of CAVs at different positions on their driving trajectories, the trajectories are discretized as shown in Figure 3, and the unit length of trajectory is $l_{1}$. Here, we denote the four road segments associated with the intersection as segments 
$a, b, c$ and $d$ in the figure. Let $P_{b c}^{n}$ denotes the $n$-th position on the path starting from road segment $b$ and ending at segment $c$, and the position when CAVs just arrive at IZ is labeled as the 0 -th position. $V_{b c, i}$ indicates the $i$-th CAV entering the intersection from segment $b$. Let $v_{b c, i}^{n}, u_{b c, i}^{n}$ and $t_{b c, i}^{n}$ denote the arrival velocity, acceleration and time to the position $P_{b c}^{n}$ for CAV $V_{b c, i}$.

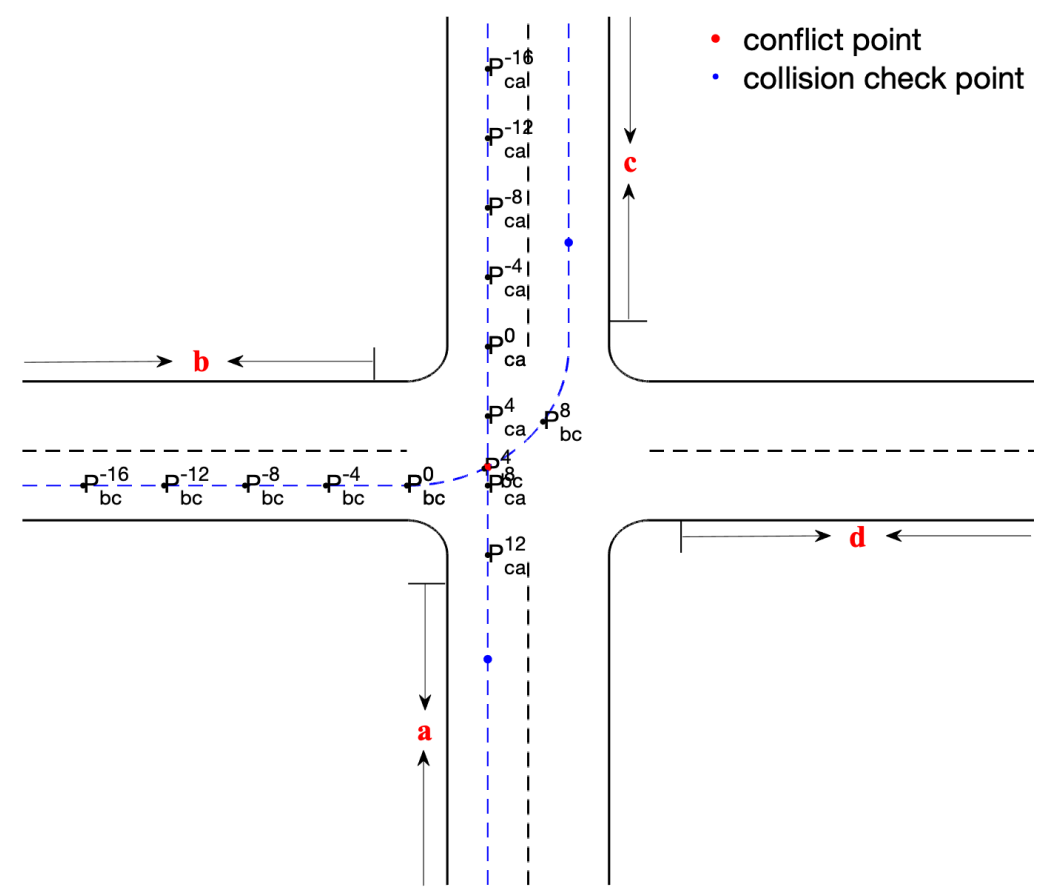

Figure 3. An illustration of discretized trajectories.

The discretized longitudinal dynamic model of $C A V V_{b c, i}$ can be described as

$$
\left\{\begin{array}{l}
v_{b c, i}^{k+1}=\sqrt{\left(v_{b c, i}^{k}\right)^{2}+2 l_{1} u_{b c, i}^{k}} \\
t_{b c, i}^{k+1}=\frac{t_{b c, i}^{k+1}-t_{b c, i}^{k}}{u_{b c, i}^{k}}+t_{b c, i}^{k}
\end{array}\right.
$$

\subsection{Vehicle Constraints}

For practical considerations, several constraints need to be imposed. This section presents the considered constraints such as safety, bounded velocities, and actuator limitations.

\subsubsection{Constraints on the Occupation Time of Conflict Points}

The conflict point is the crossing of paths, which is used to signify conflicts between CAVs in the IZ. Let $C_{b c, c a}$ denotes the conflict point formed by crossing of path $P_{b c}$ and path $P_{c a}$. In addition, assuming that $C_{b c, c a}$ is the $j$-th conflict point, which is close to position $P_{b c}^{n}$ and $P_{c a}^{m}$. Then, the occupation time of $C_{b c, c a}$ for $C A V V_{b c, i}$ and CAV $V_{c a, o}$ can be expressed as

$$
\left\{\begin{array}{l}
T_{b c, j, i}=\left(t_{b c, i}^{n-\breve{s}}, t_{b c, i}^{n-\widehat{s}}\right) \\
T_{c a, j, o}=\left(t_{c a, o}^{m-\breve{s}}, t_{c a, o}^{m-\widehat{s}}\right)
\end{array}\right.
$$

where $\breve{s}=\left\lceil\frac{l_{s}}{l_{1}}\right\rceil, \widehat{s}=\left\lceil\frac{l_{s}+l_{v}}{l_{1}}\right\rceil, l_{v}$ is the length of CAVs, and $l_{s}$ is the safe distance threshold. To guarantee that CAVs do not collide in the IZ, the occupation times of the $j$-th conflict point need to satisfy

$$
T_{b c, j, i} \cap T_{c a, j, o}=\varnothing, \forall a, b, c, i, j, o
$$




\subsubsection{Constraints on the Occupation Time in the PCZ}

Even if CAVs have passed IZ safely, there are possibilities of collision in the PCZ. For example, after a turning CAV with slow velocity has just passed IZ, a straight-going CAV that has the same exit with high velocity arrives at the PCZ. Although both CAVs can pass the IZ safely, the straight-going CAV does not have sufficient time to decelerate to avoid the conflict with the turning CAV. To avoid this kind of situation, the CAV that enters the PCZ first need to also leave the PCZ first. Assuming that $C_{b c, a c}$ is the conflict point that is close to the PCZ, which is also close to position $P_{b c}^{n}$ and $P_{a c}^{m}$. Then the collision check point is close to the position $P_{b c}^{n+s}$ and $P_{a c}^{m+s}$, where $s=\left\lceil\frac{l_{p}}{l_{1}}\right\rceil$, and $L_{p}$ is the length of the PCZ. Therefore, the occupation time in the PCZ for CAV $V_{b c, i}$ and CAV $V_{a c, o}$ can be expressed as

$$
\left\{\begin{array}{l}
T_{b c, i}=\left(t_{b c, i}^{n}, t_{b c, i}^{n+s}\right) \\
T_{a c, o}=\left(t_{a c, o}^{m}, t_{a c, o}^{m+s}\right)
\end{array}\right.
$$

To guarantee that CAVs with the same exit do not collide in the PCZ, the occupation times of PCZ need to satisfy

$$
\left(T_{b c, i} \subset T_{a c, o}\right) \cup\left(T_{b c, i} \supset T_{a c, o}\right)=\varnothing, \forall a, b, c, i, o
$$

2.3.3. Constraints on the Gap between Adjacent CAVs from the Same Approaches

To avoid collisions before CAVs drive into IZ, only after that the preceding CAV has passed position $P_{b c}^{k}(k \leq 0)$, the rear CAV can arrive at this position, which means

$$
t_{b c, i}^{k} \geq t_{b c, i-1}^{k+\widehat{s}}, k \leq 0, \forall b, c, k, i
$$

\subsubsection{Constraints on Velocity}

In the model, the straight, left-turn and right-turn velocities are limited by

$$
\left\{\begin{array}{l}
v^{\min } \leq v_{\text {straight }} \leq v_{\text {straight }}^{\max } \\
v^{\text {min }} \leq v_{\text {left }} \leq v_{\text {left }}^{\max } \\
v^{\text {min }} \leq v_{\text {right }} \leq v_{\text {right }}^{\max }
\end{array}\right.
$$

where $v_{\text {straight }}^{\max }, v_{\text {left }}^{\max }$ and $v_{\text {right }}^{\max }$ are the maximum velocity limits of CAVs when going straight, turning left and turning right, respectively. $v^{\mathrm{min}}$ is the minimum velocity of CAVs. For simplicity, we use $v_{\max \_ \text {in }}$ and $v_{\max _{-} l}$ to represent the maximum velocity limits when CAVs are in the IZ and non-IZ, respectively, which means that

$$
\begin{aligned}
& v_{\max \_ \text {in }}=\left\{\begin{array}{l}
v_{\text {straight }}^{\max } \text { go straight } \\
v_{\text {left }}^{\max }, \text { turn left } \\
v_{\text {right }}^{\max }, \text { turn right }
\end{array}\right. \\
& v_{\max \_l}=v_{\text {straight }}^{\max }
\end{aligned}
$$

\subsubsection{Constraints on Acceleration/Deceleration}

The constraints of acceleration/deceleration is denoted as

$$
u_{\min } \leq u \leq u_{\max }
$$

where $u_{\min }$ and $u_{\max }$ are the maximum deceleration and acceleration, respectively. 


\section{Scheduling Scheme}

When the occupation time of PCZ and conflict points cannot meet the constraints Equations (3) and (5), the colliding CAVs need to adopt measures to avoid collision. In this section, we proposed a priority-based avoidance rule to determine the crossing order of CAVs. As the CAVs cross the IZ with high velocity can reduce the occupancy time of conflict points and as a result improve the traffic efficiency, the CAVs should try to drive through the IZ and PCZ with as high velocities as possible. In addition, CAVs followed by emergency CAVs should pass the intersection with high priorities, to decrease the crossing delay of emergency CAVs. Therefore, based on the arrival time to the IZ and the emergency level of CAVs, we proposed a three-level priority rule to improve the traffic efficiency and shorten the delays of emergency CAVs as shown in Figure 4.

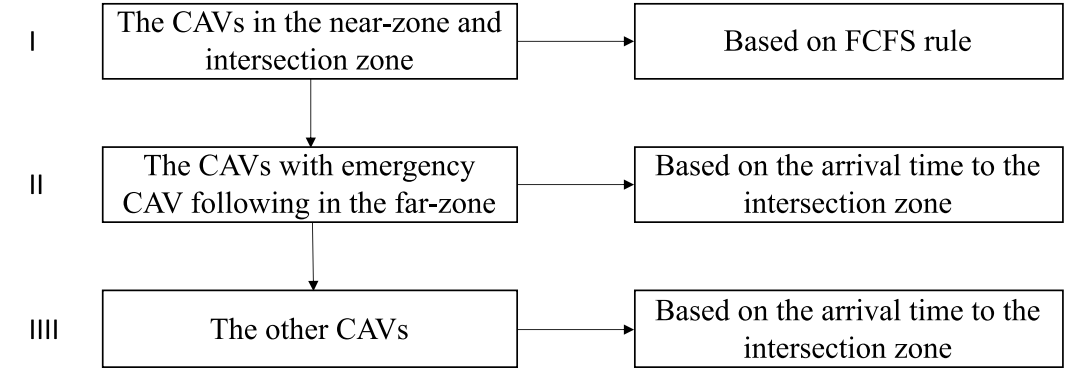

Figure 4. Priority rule.

The first level includes the CAVs in the near zone and IZ. Their priorities are determined based on the FCFS rule, which means that the CAVs driving into the near zone later need to avoid the CAVs that arrive earlier. This strategy will guarantee that the CAVs close to the IZ do not decelerate suddenly to avoid other CAVs, so as to decrease the occupation time of IZ.

The second level includes the CAVs followed by emergency CAVs in the far zone. Their priorities are determined based on the arrival time to the IZ. When an emergency CAV heads into the far zone, the priorities of the CAVs in front of this emergency CAV will be elevated to this level. Then, CAVs will reschedule their arrival time to the IZ taking into account the CAVs that have higher priorities. The new arrival time will be broadcasted by V2X communications. This strategy will guarantee that the emergency CAVs have short crossing delays.

The rest $C A V s$ will be sorted into the third level whose priorities are the lowest. The priorities of CAVs at this level are also determined based on the arrival time to the IZ. In other words, these CAVs will make sacrifices to avoid emergency CAVs and the CAVs that can arrive at IZ earlier, to improve the traffic efficiency and shorten the delays of emergency CAVs.

\section{The Decentralized Control Algorithm}

Based on the avoidance rule proposed in Section III, CAVs can make their optimal trajectory planning that meet the relevant constraints. In this section, a decentralized control algorithm is proposed to obtain optimal trajectory planning of CAVs. In particular, we set a multi-objective function, which considers the velocity, ride comfort and fuel consumption of CAVs. Then, by optimizing the multi-objective function with the penalty function method, the optimal input $\mathbf{u}^{*}$ of ego-CAV can be obtained.

\subsection{Multi-Objective Function Design}

The most critical task for intersection management is to ensure safety, which has been mentioned in the previous sections. Moreover, the performance of velocity, fuel consumption and ride comfort of CAV should also be considered. It is better for CAVs to drive through the intersection area with a short duration, low fuel consumption and good ride comfort. 


\subsubsection{The Velocity Objective}

CAVs with high velocity means low traffic delays, so CAVs should pilot with desired velocity as soon as possible. In addition, CAVs with high velocity in the IZ can reduce the occupation time of IZ, to improve the traffic efficiency of intersections. It means that high velocity in the IZ is more significant than that in other zones. Thus, we set the velocity objective as

$U_{v}=\alpha_{1} \sum_{k=-p}^{-1}\left(\frac{v_{\text {ideal_l}}-v^{k}}{v_{\text {ideal_l}} l}\right)^{2}+\alpha_{2} \sum_{k=0}^{K}\left(\frac{v_{\text {ideal_in }}-v^{k}}{v_{\text {ideal_in }}}\right)^{2}+\alpha_{1} \sum_{k=K+1}^{E}\left(\frac{v_{\text {ideal_l} l}-v^{k}}{v_{\text {ideal_l }}}\right)^{2}$

where $\alpha_{1}$ and $\alpha_{2}\left(\alpha_{1}<\alpha_{2}\right)$ are the velocity weight factors of non-IZ and IZ, and $p, K$, and $E$ are the position order of ego-CAV's current location, the last conflict point and the collision check point on its path.

\subsubsection{The Fuel Consumption Objective}

The fuel consumption of CAV is related to its velocity and acceleration. By referring to [17], the fuel consumption objective is expressed as

$$
U_{f}=\alpha_{3} \sum_{k=-p}^{E}\left[b_{0}+b_{1} v^{k}+b_{2}\left(v^{k}\right)^{2}+b_{c}\left(v^{k}\right)^{3}+u^{k}\left(c_{0}+c_{1} v^{k}+c_{2}\left(v^{k}\right)^{2}\right)\right]
$$

where $\alpha_{3}$ is the weight factor of fuel consumption, $b_{i}$ and $c_{i}(i=0,1,2)$ are the coefficients to calculate fuel consumption.

\subsubsection{The Ride Comfort Objective}

The ride comfort of CAV is mainly related to its acceleration/deceleration and the fluctuation of acceleration/deceleration. The ride comfort objective is described as

$$
U_{c}=\alpha_{4} \sum_{k=-p}^{E}\left(\Delta u^{k}\right)^{2}+\alpha_{5} \sum_{k=-p}^{E}\left(u^{k}\right)^{2}
$$

where $\Delta u^{k}=u^{k}-u^{k-1}$ is the fluctuation of acceleration/deceleration, $\alpha_{4}$ and $\alpha_{5}$ are the ride comfort weight factors.

In summary, the multi-objective function for ego-CAV can be expressed as

$$
\begin{aligned}
& \min _{\mathbf{u}} J=U_{v}+U_{f}+U_{v} \\
& \text { s.t.(1), (3), (5), (6), (7), (9) }
\end{aligned}
$$

\subsection{Penalty Function Method}

To obtain the optimal input $\mathbf{u}^{*}$ of CAV, we adopted the penalty function method to optimize the multi-objective function as described by Equation (13). Assuming there are $M$ CAVs whose priorities are higher than that of CAV $V_{b c, i}$, among which there are $N(M \geq N)$ CAVs that have the same exit with CAV $V_{b c, i}$. Let $T_{a d, j, o}^{m}$ represent the $m$-th CAV's occupation time of the $j$-th conflict point, and let $T_{a c, 0}^{n}$ represent the $n$-th CAV's occupation time of PCZ. The constraints can be transferred as penalty items, which is described as 


$$
\begin{aligned}
p(\mathbf{u})= & \mu_{1} \sum_{k=-p}^{E}\left(\min \left[0,\left(u_{\max }-u^{k}\right)\left(u^{k}-u_{\min }\right)\right]\right)^{2}+ \\
& \mu_{2} \sum_{k=-p}^{-1}\left(\min \left[0,\left(v_{\max \_}-v^{k}\right)\left(v^{k}-v_{\min }\right)\right]\right)^{2}+ \\
& \mu_{2} \sum_{k=0}^{K}\left(\min \left[0,\left(v_{\max \_i n}-v^{k}\right)\left(v^{k}-v_{\min }\right)\right]\right)^{2}+ \\
& \mu_{2} \sum_{k=K+1}^{E}\left(\min \left[0,\left(v_{\max \_l}-v^{k}\right)\left(v^{k}-v_{\min }\right)\right]\right)^{2}+ \\
& \mu_{3} \sum_{n=1}^{N}\left(\min \left[0,\left(\widehat{T}_{b c, i}-\widehat{T}_{a c, o}^{n}\right)\left(\breve{T}_{b c, i}-\breve{T}_{a c, o}^{n}\right)\right]\right)^{2}+ \\
& \mu_{3} \sum_{k=-p}^{-1}\left(\min \left[0,\left(t_{b c, i}^{k}-t_{b c, i-1}^{k+\widehat{s}_{1}}\right)\right]\right)^{2}+ \\
& \mu_{3} \sum_{m=1}^{M} \sum_{j=1}^{N_{1}}\left(\min \left[0,\left(\widehat{T}_{b c, j, i}-\breve{T}_{a d, j, o}^{m}\right)\left(\breve{T}_{b c, j, i}-\breve{T}_{a d, j, o}^{m}\right)\right]\right)^{2}
\end{aligned}
$$

where $\mu_{i}(i=1,2,3)$ is the penalty factor. $\breve{T}_{b c, i}$ and $\widehat{T}_{b c, i}$ are the left and right endpoints of the interval $T_{b c, i} \cdot \breve{T}_{b c, j, i}$ and $\breve{T}_{b c, j, i}$ are the left and right endpoints of the interval $T_{b c, j, i}$. $N_{1}=20$ is the number of conflict points. Therefore, the multi-objective function with constraints can be transferred as a multi-objective function without constraints

$$
\min _{\mathbf{u}} \tilde{J}=J(u)+p(u)
$$

Then, by optimizing Equation (15), the optimal input $\mathbf{u}^{*}$ of ego-CAV can be obtained.

\section{Simulation Results}

In this section, simulation is performed with MATLAB. And the centralized reservation scheme [12], the decentralized MPC-based scheme [13] and the traditional traffic light scheme are presented to compare with the proposed AIM scheme. In the simulation, the arrival rate of CAVs at each lane is assumed to be a Poisson process with the same average value $\lambda$. In addition, 1/2,1/4 and 1/4 of CAVs will go straight, turn right, and turn left at the intersection for each lane, respectively. Assuming that CAVs pilot with traffic-flow model [18] before arriving at the intersection area. In addition, each CAV need to share its information periodically including emergency level, size, predicted occupation time of conflict points and PCZ etc. Then, CAVs can determine the crossing order according to the proposed priority rule and optimize their trajectory planning according to the proposed control algorithm periodically. As the total number of CAVs is fixed within given simulation time, the traffic efficiency and traffic delays can be determined for each scheme. The model parameters are set according to practical considerations, which are listed in Table 1 in detail. 
Table 1. Simulation parameters.

\begin{tabular}{cc}
\hline Parameter & Value \\
\hline Vehicle Length $l_{v}$ & $4 \mathrm{~m}$ \\
\hline Safety Length $l_{s}$ & $3 \mathrm{~m}$ \\
\hline Lane Width & $4 \mathrm{~m}$ \\
\hline Intersection Area & $300 * 300 \mathrm{~m}^{2}$ \\
\hline Far zone length $L_{f}$ & $240 \mathrm{~m}$ \\
\hline Near zone length $L_{n}$ & $54 \mathrm{~m}$ \\
\hline IZ length $L_{i}$ & $12 \mathrm{~m}$ \\
\hline PCZ length $L_{p}$ & $19 \mathrm{~m}$ \\
\hline Maximum Velocity of Going Straight $v_{\text {straight }}^{\max }$ & $15 \mathrm{~m} / \mathrm{s}$ \\
\hline Maximum Velocity of Turning Left $v_{\text {left }}^{\max }$ & $8 \mathrm{~m} / \mathrm{s}$ \\
\hline Maximum Velocity of Turning Right $v_{\text {right }}^{\max }$ & $4 \mathrm{~m} / \mathrm{s}$ \\
\hline Maximum Deceleration $a_{\min }$ & $-6 \mathrm{~m} / \mathrm{s}^{2}$ \\
\hline Maximum Acceleration $a_{\max }$ & $3 \mathrm{~m} / \mathrm{s}^{2}$ \\
\hline Simulation Time & $5 \mathrm{~min}$ \\
\hline
\end{tabular}

In Figure 5, we compare the traffic efficiency under different schemes for different traffic loads. As we can see, the traffic efficiency of the proposed scheme is similar to that of the centralized reservation scheme, and the maximum traffic efficiency of the proposed scheme can be increased by about $70 \%$ as compared with that of the decentralized MPCbased scheme. This demonstrates that our proposed scheme can achieve high traffic efficiency. This is because that the proposed scheme guarantees that the CAVs can drive through the IZ with high velocity and the CAVs whose arrival time to the IZ are earlier will be assigned with higher crossing priorities.

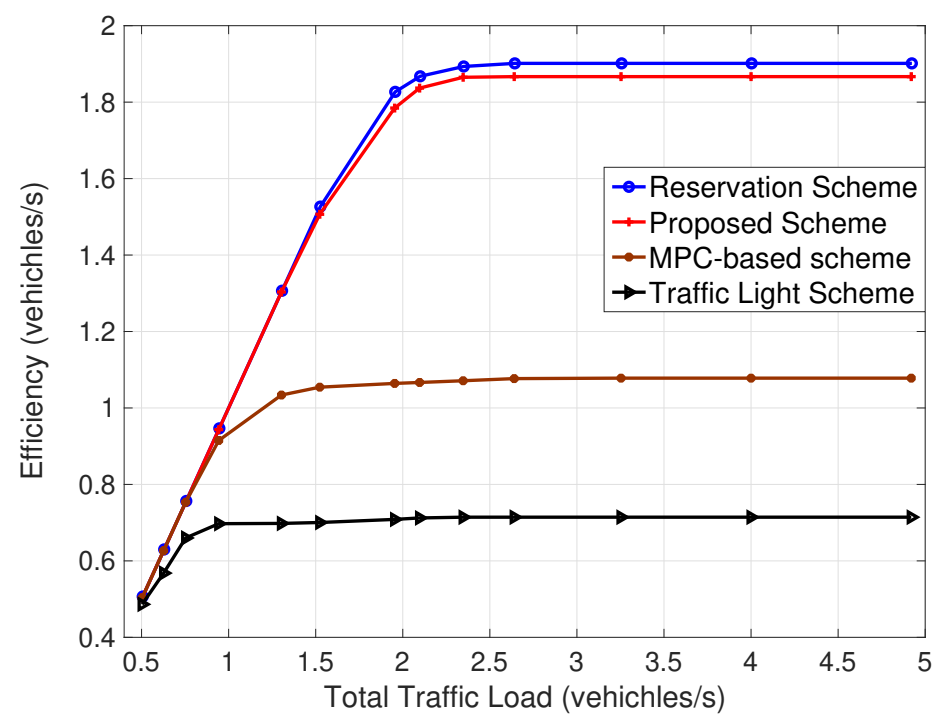

Figure 5. Traffic efficiency comparison under different schemes.

Figure 6 shows the average delay of the passed CAVs at different total traffic load under different schemes. It can be seen that the average delay will increase as the traffic load increases, and the average delay of the proposed scheme is slightly bigger than that of the reservation scheme and much smaller than that of the MPC-based scheme. This is in accordance with the traffic efficiency as shown is Figure 5. 
Figure 7 shows that the maximum delay of the passed CAVs at different total traffic load under different schemes. It can be seen that the maximum delay of the passed CAVs will increase as the traffic load increases. In addition, our proposed scheme has lower maximum delay compared with the other schemes, which means that the proposed scheme has better crossing fairness. This is because that the crossing order of the proposed scheme is mainly determined by the arrival time to IZ other than the FCFS rule. Thus, the CAVs that drive into intersection area at a later time but can arrive at IZ earlier could be assigned with higher crossing priorities.

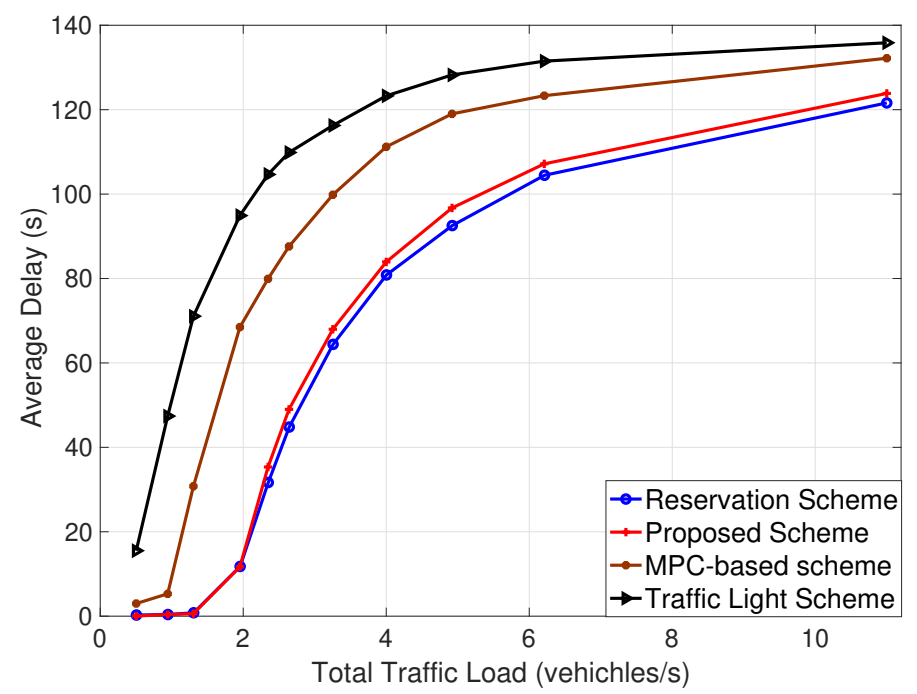

Figure 6. Average delay comparison under different schemes.

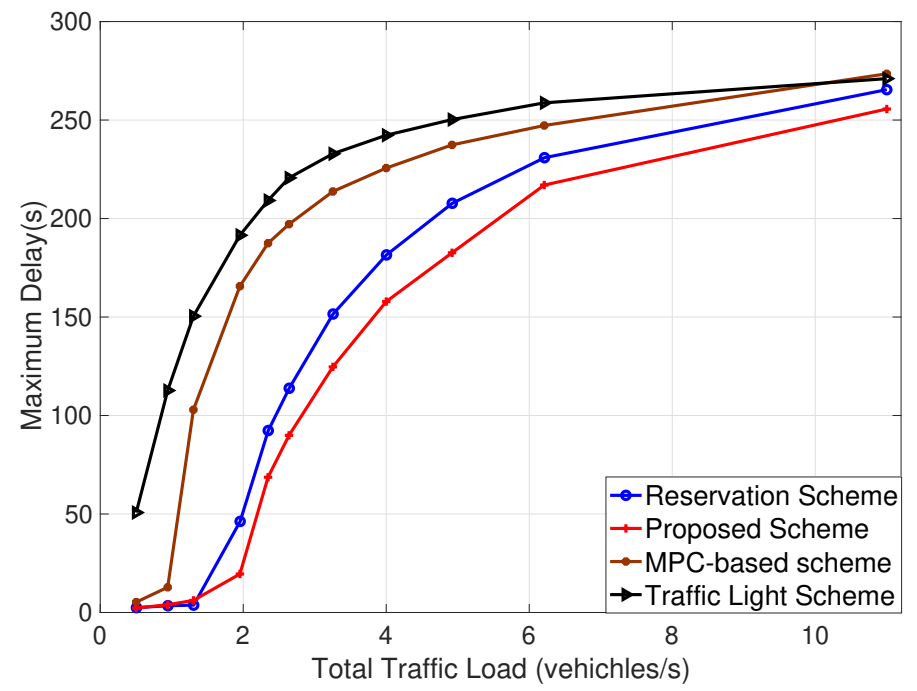

Figure 7. Maximum delay comparison under different scheme.

To demonstrate the superiority of the emergency CAVs in the proposed scheme, the delays of the emergency CAVs with different arrival time to the intersection area are presented in Figure 8. As we can see, when these emergency CAVs are labeled in our proposed scheme, they have shorter delays than when they are not labeled, which verifies that our proposed scheme can handle the emergency CAVs with higher priorities. The reason is that the CAVs followed by emergency CAVs in the far zone are assigned with higher crossing priorities in our proposed scheme. 


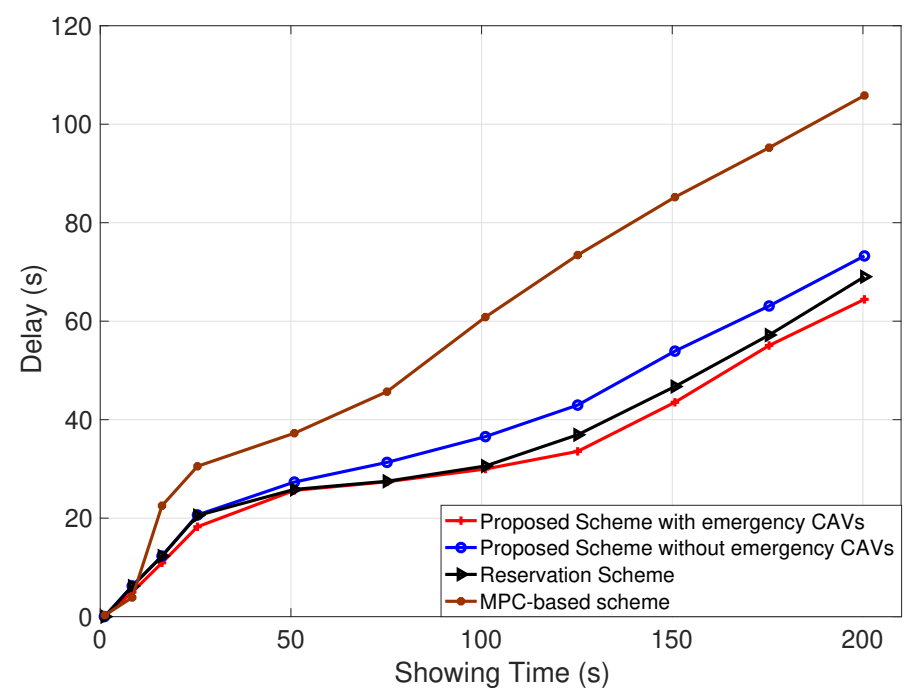

Figure 8. Delay of emergency CAVs under different schemes.

\section{Conclusions and Outlook}

In this paper, we investigated the autonomous intersection management problem with CAVs. To this end, we first proposed a novel intersection-vehicle model, which can consider all possible collisions of CAVs in the intersection area. Then, to avoid conflicts between $\mathrm{CAVs}$, a priority-based avoidance rule was presented to improve the traffic efficiency and emergency CAVs delays. Finally, based on the model and the avoidance rule, we set a multi-objective function to design the optimal trajectories of CAVs. Through extensive simulations, the performance of the proposed scheme was compared with the centralized reservation scheme, the decentralized MPC-based scheme and the traffic light scheme. Simulation results verified that our proposed scheme can achieve good performance in terms of both traffic efficiency and flexibility in shortening the delays of emergency CAVs.

This article only showed that the proposed decentralized scheme has better performance than some other schemes by simulations with given model parameters. As CAVs cross the intersection with high velocity in the proposed scheme, potential collisions could happen by any deviations from the model assumption in realistic scenarios. Thus, as the next important step, the parameter values need to be further explored to make sure that CAVs can cross the intersection area even with minor errors. Moreover, It is also an interesting topic to extend this scheme to the scenarios of multiple intersections and mixed traffics of autonomous and human-driven vehicles.

Author Contributions: Conceptualization, H.Z. and R.Z.; methodology, D.D. and X.C.; software, C.C. and L.Y.; validation, H.Z., D.D. and R.Z.; formal analysis, X.C.; investigation, L.Y.; data curation, H.Z.; writing-original draft preparation, H.Z.; writing—review and editing, D.D. and R.Z.; supervision, X.C. All authors have read and agreed to the published version of the manuscript.

Funding: This research was partially funded by the Ministry National Key Research and Development Project under Grant 2017YFE0121400, Guangdong Key R\&D Project under Grant 2019B010153003, and the National Science Foundation under Grants CNS-2103256 and CNS-1932139.

Institutional Review Board Statement: Not applicable.

Informed Consent Statement: Not applicable.

Data Availability Statement: Not applicable.

Conflicts of Interest: The authors declare no conflict of interest. 


\section{References}

1. Schrank, B.; Eisele, B.; Lomax, T.; Bak, J. 2015 Urban Mobility Scorecard; Texas A\&M Transportation Institute: Bryan, TX, USA, 2015.

2. Qian, Y.; Wang, C.; Wang, H.; Wang, Z. The optimization design of urban traffic signal control based on three swarms cooperative particle swarm optimization. In Proceedings of the 2007 IEEE International Conference on Automation and Logistics, Jinan, China, 18-21 August 2007; pp. 512-515.

3. He, Z.; Wang, L.; Li D.; Zhang, L. Steady-state signal control for urban traffic networks. In Proceedings of the 2015 IEEE 18 th International Conference on Intelligent Transportation Systems, Las Palmas, Spain, 15-18 Septemper 2015; pp. 463-470.

4. Wang, N.; Wang, X.; Palacharla, P.; Ikeuchi,T. Cooperative autonomous driving for traffic congestion avoidance through vehicle-tovehicle communications. In Proceedings of the 2017 IEEE Vehicular Networking Conference, Torino, Italy, 27-29 November 2017; pp. 327-330.

5. Xu, L.; Yin, G.; Zhang, N. Flocking cooperative driving control of four-wheel independently driving electric autonomous vehicles considering vehicular dynamic processes. In Proceedings of the 2016 35th Chinese Control Conference, Chengdu, China, 27-29 July 2016; pp. 4487-4492.

6. Wang, B.; Zhang, R.; Chen, C.; Cheng, X.; Yang, L.; Jin, Y. Interference hypergraph-based 3D matching resource allocation protocol for NOMA-V2X networks. IEEE Access 2019, 7, 90789-90800.

7. Li, B.; Zhang, Y.; Feng, Y.; Zhang, Y.; Ge, Y.; Shao, Z. Balancing computation speed and quality: A decentralized motion planning method for cooperative lane changes of connected and automated vehicles. IEEE Trans. Intell. Veh. 2018, 3, 340-350.

8. Lee, C.; Fumagalli, A. Internet of things security-multilayered method for end to end data communications over cellular networks. In Proceedings of the 2019 IEEE 5th World Forum on Internet of Things, Limerick, Ireland, 15-18 April 2019; pp. 24-28.

9. Zhu, L.; Yu, F. R. Wang, Y.; Ning, B.; Tang, T. Big data analytics in intelligent transportation systems: A survey. IEEE Trans. Intell. Transp. Syst. 2019, 20, 383-398.

10. Au, T.; Fok, C.; Vishwanath, S.; Julien, C.; Stone, P. Evasion planning for autonomous vehicles at intersections. In Proceedings of the 2012 IEEE/RSJ International Conference on Intelligent Robots and Systems, Vilamoura, Portugal, 7-12 October 2012; pp. 1541-1546.

11. Rios-Torres, J.; Malikopoulos, A. A. A survey on the coordination of connected and automated vehicles at intersections and merging at highway on-ramps. IEEE Trans. Intell. Transp. Syst. 2017, 18, 1066-1077.

12. Dresner, K.; Stone, P. A multiagent approach to autonomous intersection management. J. Artif. Intell. Res. 2008, 31, 591-656.

13. Zhao, X.; Wang, J.; Yin, G.; Zhang, K. Cooperative driving for connected and automated vehicles at non-signalized intersection based on model predictive control. In Proceedings of the 2019 IEEE Intelligent Transportation Systems Conference, Auckland, New Zealand, 27-30 October 2019; pp. 2121-2126.

14. Makarem, L.; Gillet, D. Fluent coordination of autonomous vehicles at intersections. In Proceedings of the 2012 IEEE International Conference on Systems, Man, and Cybernetics, Seoul, Korea, 14-17 October 2012; pp. 2557-2562.

15. Ali, H.; Khaled, S.; Shehata, O.; Morgan, E. Decentralized intersection management of autonomous vehicles using nonlinear MPC. In Proceedings of the 2020 2nd Novel Intelligent and Leading Emerging Sciences Conference, Giza, Egypt, 24-26 October 2020; pp. 410-415.

16. Wei, H.; Mashayekhy, L.; Papineau, J. Intersection management for connected autonomous vehicles: A game theoretic framework. In Proceedings of the 2018 21st International Conference on Intelligent Transportation Systems, Maui, HI, USA, 4-7 November 2018; pp. 583-588.

17. Kamal, M.; Mukai, M.; Murata, J.; Kawabe, T. Model predictive control of vehicles on urban roads for improved fuel economy. IEEE Trans. Control Syst. Technol. 2013, 21, 831-841.

18. van Arem, B.; van Driel, C.; Visser, R. The impact of cooperative adaptive cruise control on traffic-flow characteristics. IEEE Trans. Intell. Trans. Syst. 2006, 7, 429-436. 